\title{
TUNING POSSIBILITIES OF THE LONGITUDINAL BEAM SHAPE OF A RACETRACK MICROTRON
}

\author{
W.H.C. Theuws, J.I.M. Botman, H.L. Hagedoorn, A.F.J. Hammen, \\ Eindhoven University of Technology, Cyclotron Laboratory, \\ P.O. Box 513, 5600 MB Eindhoven, The Netherlands.
}

\begin{abstract}
The beam shape in the longitudinal phase plane at the extraction point of an RTM strongly depends on the choice of the stable phase of the accelerating cavity. This is due to the fact that the main longitudinal focusing force is the derivative of the accelerating potential with respect to time (or phase). Furthermore, this focusing force is applied to the electron beam each turn, such that the output beam shape in the longitudinal phase plane is significantly affected. This effect can for instance be used in order to minimise the bunch length or the energy spread of the output beam of the RTM. For this paper we have used the lay-out of the Eindhoven RTM for the calculations of the stable-phase variations, which have been studied in linear approximation. The stable phase area for this RTM equals 18 degrees. It will be shown that stable-phase variations in the order of 1 degree already have a significant impact on the longitudinal beam shape. Furthermore, some remarks are made on the consequences of non-linear beam dynamics in relation to stable-phase variations.
\end{abstract}

\section{BUNCH-LENGTH COMPRESSION}

In contrast to linear accelerators the shape of an RTM's output beam in the longitudinal phase plane can be modified. This can be done by changing the amplitude and phase of the RTM cavity. The possibility to modify this beam shape makes it possible to either minimise the bunch length or the energy spread of the output beam [1].

The energy gain per turn, $E_{r}$, has been chosen $5 \mathrm{MeV}$ for the Eindhoven racetrack microtron. As RF acceleration is being used this energy gain $E_{r}$ is set as

$$
E_{r}=\hat{E} \cos \left(\phi_{s}\right) \text {, }
$$

where $\hat{E}$ is the amplitude and $\phi_{s}$ the stable phase of the cavity voltage. The slope of the RF voltage at the stable phase provides the longitudinal focusing force. For that reason it is obvious that the choice of the combination of $\hat{E}$ and $\phi_{s}$ has an influence on the longitudinal beam shape of the RTM's output beam. As this focusing force is applied 13 times to the electron beam it can be expected that a slightly different choice of $\hat{E}$ and $\phi_{s}$ may have a great impact.
First, this impact has been studied, utilising linear motion only, see section 2 . Second, the validity of the linear theory in the RTM is discussed in section 3, where the linearity of the longitudinal phase plane is studied by means of a numerical simulation program of the Eindhoven RTM. All calculations, which are presented in this paper, start just before the first cavity traversal. The beam shape in the longitudinal phase plane just before the first cavity traversal has been estimated from measurements and simulations of the RTM injector [2]. This beam shape can be approximated by an ellipse with Twiss-parameters: $\alpha=0, \beta=0.185 \mathrm{~m}, \gamma=5.41 \mathrm{~m}^{-1}$, and $\varepsilon$ $=2 \cdot 10^{-6} \mathrm{~m}$. This ellipse is used as input for the first-order calculations in section 2 and also for some of the numerical calculations in section 3 .

\section{FIRST-ORDER CALCULATIONS}

The effect of the stable phase of the cavity, $\phi_{s}$, on the beam shape in the longitudinal phase plane has been studied in linear approximation. The transfer matrix, $\mathbf{M}_{n}$, describing the transport of the $n^{\text {th }}$ orbit in the racetrack microtron starting just before the cavity is given by the product of first-order matrices:

$$
\begin{aligned}
& \mathbf{M}_{n}=\mathbf{D}_{1 / 2(L-L c a v)} \cdot \mathbf{F}_{\alpha_{1}} \cdot \mathbf{B}_{1, n} \cdot \mathbf{F}_{\alpha_{2}} \cdot \mathbf{B}_{2, n} \cdot \mathbf{F}_{\alpha_{3}} \cdot \mathbf{D}_{L+2 L} \tau_{\tau_{n}} \cdot \\
& \mathbf{F}_{\alpha_{3}} \cdot \mathbf{B}_{2, n} \cdot \mathbf{F}_{\alpha_{2}} \cdot \mathbf{B}_{1, n} \cdot \mathbf{F}_{\alpha_{1}} \cdot \mathbf{D}_{1 / 2(L-L a v)} \cdot \mathbf{C}_{\hat{E}, \phi_{s}} .
\end{aligned}
$$

The transport that is described by all the matrices in this equation is pointed out in figure 1 . The matrices $\mathbf{D}, \mathbf{B}, \mathbf{F}$,

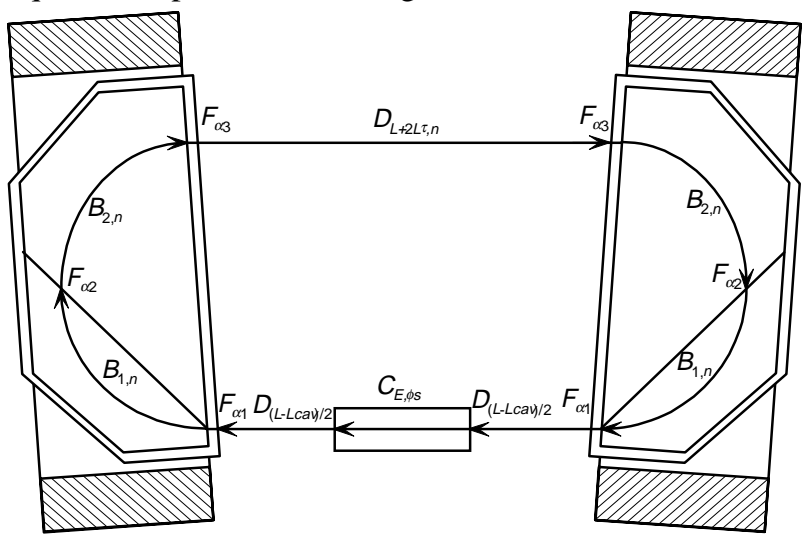

Figure 1: The transport described by the matrices for the linear approximation. 


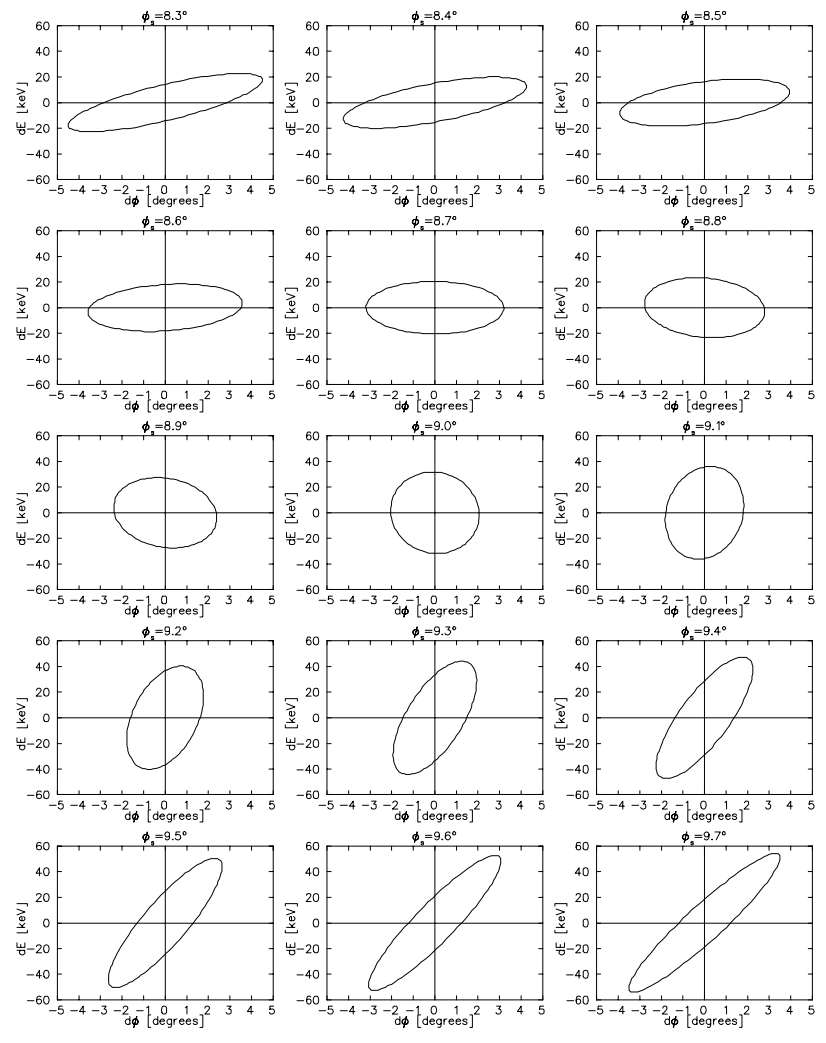

Figure 2: The beam shape in the longitudinal phase space at the extraction point of the RTM for different values of $\phi_{s}$.

$\mathbf{C}$ in eq.(2) describe the effect of a drift space, a sector bending magnet, a non-normal entrance/exit of a magnet and an accelerating cavity, respectively. The subscripts 1 and 2 in $\mathbf{B}_{1, n}$ and $\mathbf{B}_{2, n}$ refer to the sectors 1 and 2 of the main bending magnets, respectively. The subscripts $\alpha_{1}, \alpha_{2}$ and $\alpha_{3}$ denote the entrance/exit angles for the edge focusing. The parameters $\hat{E}$ and $\phi_{s}$ are the amplitude and stable phase of the cavity potential. $L_{c a v}$ is the length of the cavity, $L$ the distance between the bending magnets at the central axis of the cavity, and $L_{\tau}$ is the extra drift that results from the rotation of the main bending magnet over $\tau$. The subscript $n$ indicates that the concerning matrix is orbit dependent. The matrix that describes the transport of all twelve orbits, denoted by $\mathbf{M}$ is given by the product of matrices $\mathbf{M}_{12}$ through $\mathbf{M}_{1}$.

The longitudinal sub-matrix of $\mathbf{M}$ together with the longitudinal input beam have been used to calculate the longitudinal output beam of the RTM for different values of $\phi_{s}$. The results are shown in figure 2. From this figure it appears that variations in $\phi_{s}$ that are much smaller than the stable phase area of about 18 degrees have a large influence on the beam shape in the longitudinal phase space. This implies that it is possible to use $\phi_{s}$ to influence the longitudinal beam shape, such that this beam shape matches the acceptance of the following electron-optical
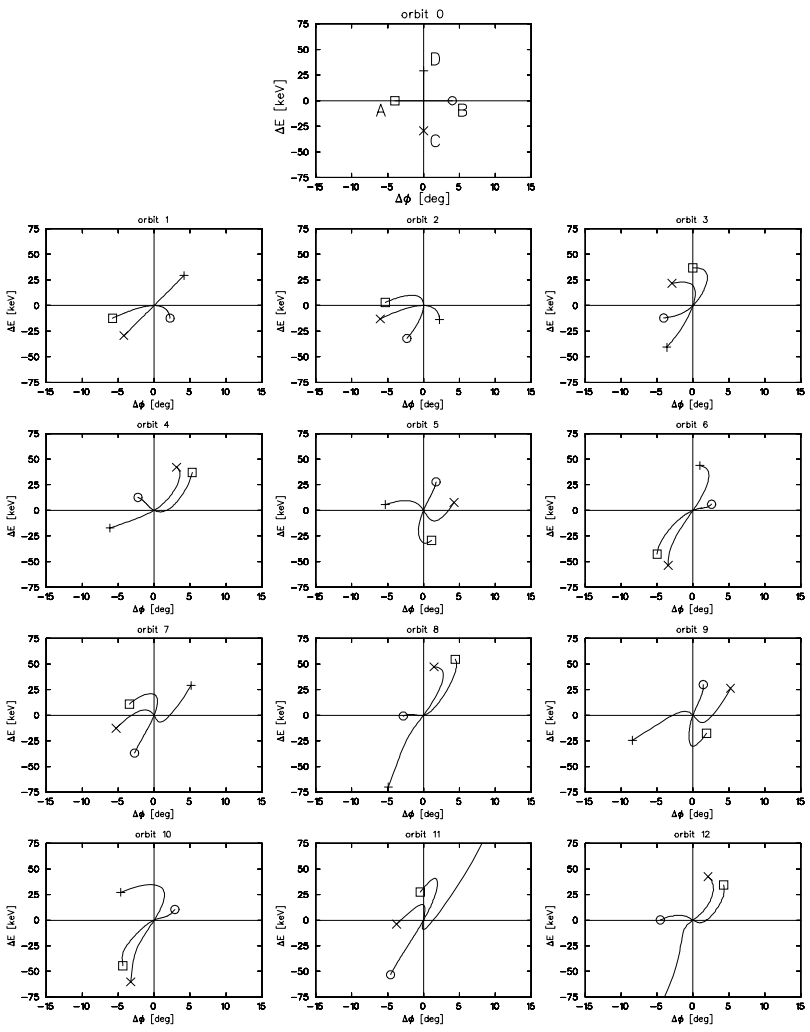

Figure 3: Two straight lines in the longitudinal phase plane $\mathrm{AB}$ and $\mathrm{CD}$ are injected just before the first cavity traversal (orbit 0 ). The stable phase of the cavity has been set to 9 degrees. These lines are given after each full orbit.

system better that it might have done with the nominal settings for $\hat{E}$ and $\phi_{s}$.

\section{VALIDITY OF THE LINEAR THEORY}

In order to verify the validity of the linear theory, two straight line segments in the longitudinal phase plane, which are a little larger than the main axes of the nominal longitudinal input beam as it is delivered by the RTM injector, have been used as input for numerical tracking through the Eindhoven RTM, see figure 3. From this figure it can be seen that the longitudinal sizes of the linac beam are much larger than the linear regime. Therefore, the longitudinal input beam has also been calculated through the Eindhoven RTM by means of the numerical simulation program for different values of $\phi_{s}$, see figure 4 . Of course, the longitudinal beam shape still depends on $\phi_{s}$, but making the bunch length shorter or the energy spread smaller by means of $\phi_{s}$ is less effective. On the other hand, if the RTM will be used for the acceleration of short bunches [3], another pre-accelerator, which will already produce much shorter bunches, will become necessary. In that case the linear approximation may become valid and useful. 

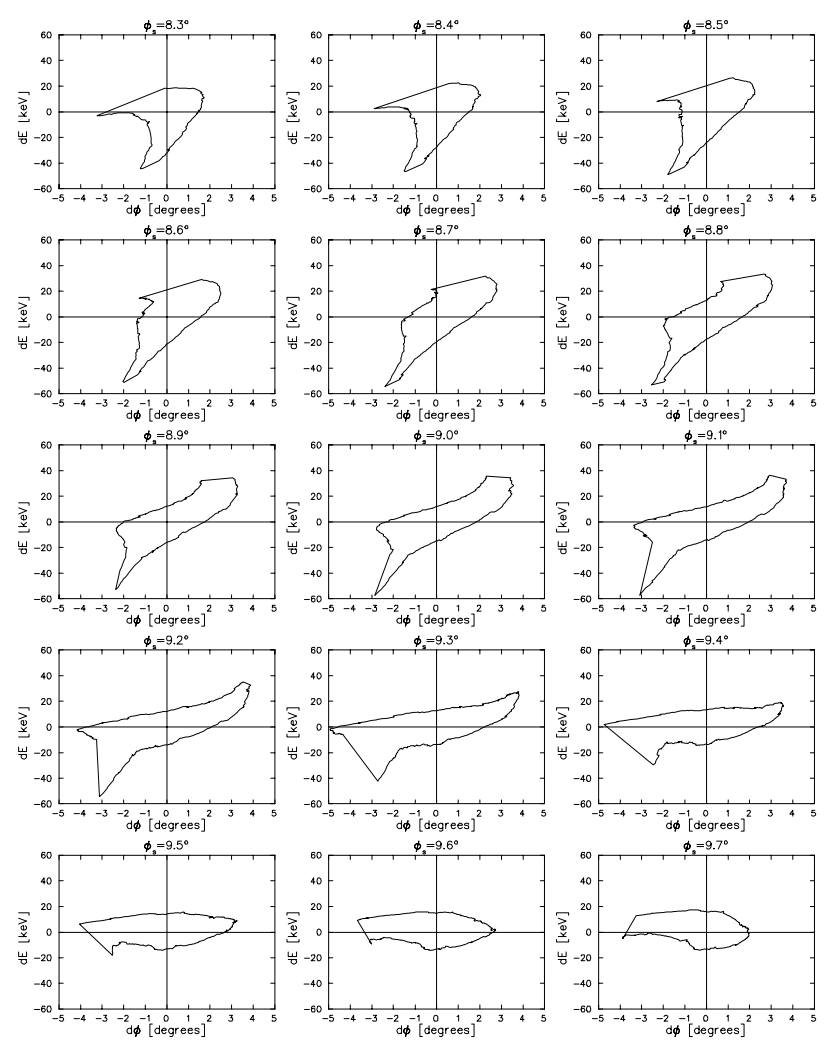

Figure 4: The longitudinal beam shape of the RTM output beam for different values of $\phi_{s}$.

As an example a beam with the nominal longitudinal beam shape but with a much smaller longitudinal emittance of $\varepsilon=1 \cdot 10^{-7} \pi \mathrm{m}$ has been tracked through the RTM numerically, with $\phi_{s}=9$ degrees. The longitudinal output beam is shown in figure 5 . This longitudinal beam shape is similar to the beam shape shown in figure 2 for $\phi_{s}=8.3$ degrees. Now, say that it is tried to minimise the energy-spread of the beam. Then it can be seen form figure 2 that this is achieved for $\phi_{s}=8.7$ degrees, which is 0.4 degrees above the 8.3 degrees representing the situation we have in our experiment for 9.0 degrees. So, adding 0.4 degrees to the 9.0 degrees gives the output beam which is also shown in figure 5 . In this figure the energy spread is minimised quite well.

\section{CONCLUDING REMARKS}

The RTM's output-beam shape in the longitudinal phase plane is very sensitive to small variations of the stable phase of the RTM's accelerating cavity. Therefore, the choice of this stable phase can be used to minimise either the bunch length or the energy spread of the output beam. The principle has been studied in linear approximation, but it has been shown that the linear approximation is only valid for longitudinal beam sizes that are much smaller than the longitudinal beam size that is delivered by the current injector. The non-linear behaviour of the RTM makes this minimisation less effective, but still
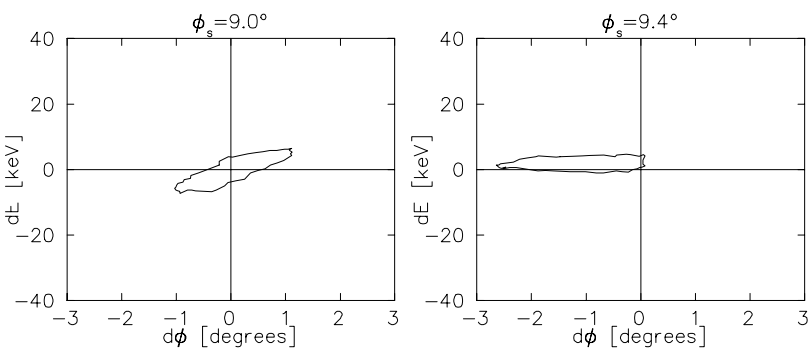

Figure 5: The longitudinal output beam shape for a beam that is injected with a small longitudinal emittance $\left(\varepsilon=1 \cdot 10^{-7} \pi \mathrm{m}\right)$ for $\phi_{s}=9.0$ degrees and $\phi_{s}=9.4$ degrees.

useful, as has been shown. Furthermore, if short bunches will have to be accelerated with the RTM, another injector will be used for the microtron. This injector will most-probably produce beams with smaller longitudinal emittances, such that the linear approximation might become valid, which makes the minimisation of the bunch length of the energy spread more effective.

If short bunches are to be accelerated in the RTM other problems, which have not been considered in this paper at all, may play an important role. For instance Coherent Synchrotron Radiation, which is important if short bunches are bent by a magnetic field, may destroy the short bunch length and therefore the RTM may not be useful as an accelerator for ultra-short bunches at all [3].

\section{REFERENCES}

[1] W.H.C. Theuws, J.I.M. Botman, H.L. Hagedoorn, The Eindhoven linac-racetrack microtron combination, Nucl. Instr. and Meth. B 139 (1998) $522-526$.

[2] A.F.J. Hammen, Particle dynamics in the $10 \mathrm{MeV}$ TUE electron linac, Internal report VDF/NK 97-07, M.Sc. Thesis, Eindhoven University of Technology (1997).

[3] M.J. van der Wiel, Personal communications (1998). 